\title{
Faire la cuisine dans les familles grecques de Venise au XVII ${ }^{\mathrm{e}}$ siècle
}

Témoignages d'archives sur les ustensiles de cuisine, de rangement et de service

Cooking in the Greek Households of 17th century Venice: Ustensils and Vessels for Cooking, Storage and Serving, Presented through Archival Evidence

\section{Ourania Karagianni}

\section{OpenEdition}

\section{Journals}

Édition électronique

URL : https://journals.openedition.org/ceb/7242

DOI : 10.4000/ceb.7242

ISSN : 2261-4184

Éditeur

INALCO

Édition imprimée

ISBN : 978-2-85831-230-6

ISSN : 0290-7402

\section{Référence électronique}

Ourania Karagianni, «Faire la cuisine dans les familles grecques de Venise au xvıe siècle », Cahiers

balkaniques [En ligne], Hors-série | 2016, mis en ligne le 20 mars 2017, consulté le 06 juillet 2021. URL : http://journals.openedition.org/ceb/7242 ; DOI : https://doi.org/10.4000/ceb.7242

Ce document a été généré automatiquement le 6 juillet 2021.

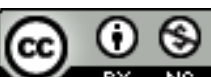

Cahiers balkaniques est mis à disposition selon les termes de la Licence Creative Commons Attribution - Pas d'Utilisation Commerciale 4.0 International. 


\section{Faire la cuisine dans les familles grecques de Venise au XVII ${ }^{\mathrm{e}}$ siècle}

Témoignages d'archives sur les ustensiles de cuisine, de rangement et de service

Cooking in the Greek Households of 17th century Venice: Ustensils and Vessels

for Cooking, Storage and Serving, Presented through Archival Evidence

\section{Ourania Karagianni}

1 Commençons par un voyage imaginaire à Venise, où des Grecs ont trouvé refuge après la prise de Constantinople en 1453 par les Ottomans. Plus précisément, une visite dans les quartiers autour de la place des Grecs, le Campo dei Greci, lieu de vie de la communauté grecque depuis le XVI ${ }^{\mathrm{e}}$ siècle (MAVROIDI, 1976). L'ensemble architectural du Campo dei Greci, qui regroupe l'église orthodoxe de Saint-Georges, la Scoletta, siège de la confrérie grecque et le Collège Flanginien, assure, en tant que centre de développement, l'image publique de la population grecque de la ville (MALTEzou, 1999). C'est ici où l'on voit se déployer le pouvoir du culte orthodoxe, l'administration, la représentation officielle devant l'État vénitien, et l'enseignement dispensé (MALTEZou, 1999B). Il convient de noter que l'étude de témoignages d'archives, une source précieuse pour ce qui est de la forte présence grecque à Venise, nous éclaire sur de nombreux aspects de leur vie publique et privée tels que les activités professionnelles, le statut social, les aspects identitaires et idéologiques (MALTEZou, 2012, 131-161), la définition et l'évolution de l'esthétique du goût (TSELENTI-PAPADOPoulou, 1998, 89-95; malteZou, 2001 ; TSELENTI-PAPADOPoulou, 2004, 459-471). C'est par le biais de contrats de mariage, de testaments et d'autres documents notariés que l'on voit émerger un tableau de la vie familiale tandis que les inventaires après décès, les registres détaillés des biens mobiliers ${ }^{1}$, nous informent sur la vie quotidienne des foyers grecs, pauvres ou prospères.

2 Les objets enregistrés dans les cuisines des ménages grecs de Venise au cours du $\mathrm{XVII}^{\mathrm{e}}$ siècle, sont révélateurs de leur culture matérielle et alimentaire. Le résultat de mon étude est fondé sur la consultation d'archives, de testaments et d'enregistrements 
de mobiliers des Grecs de Venise puisés dans les Archives d'État de la ville aussi bien que dans les Archives de l'Institut hellénique d'études byzantines et post-byzantines de la Sérénissime (MALTEZou, 2008). Plus précisément, dans un premier temps, ces objets sont étudiés en tant que témoignage médiat des heures consacrées dans la vie quotidienne à stocker, à traiter et à cuisiner les denrées afin de préparer les repas (LATRÉMOLIÈRE \& QUELLIER, 2012, 68-95). Dans un deuxième temps, on étudie leur rapport avec le rituel de consommation, moment privilégié de rassemblement et de détente familiale (FORTINI BROWN, 2005, 144-157; AJMAR-WOLLHeIM, 2006, 206-214). Ce qui nous intéresse est comment ce mobilier culinaire et d'autres objets d'apparat plus ou moins simples, associés à la présentation et à la consommation de mets, nous renseignent d'une part, sur l'univers matériel des Grecs de Venise et d'autre part sur le brassage des pratiques alimentaires.

3 Ce sont les témoignages d'archives, et surtout les inventaires de biens mobiliers, qui nous permettent de restituer l'environnement domestique moyen de Venise: le notaire, ayant répertorié les biens mobiliers au portego, - l'espace d'accueil principal ainsi que dans les chambres à coucher, se dirigeait vers la cuisine et les espaces auxiliaires tels que les entrepôts, les blanchisseries, etc. La cheminée, véritable symbole de l'espace cuisine, la crémaillère et le matériel en métal complémentaire sont les éléments essentiels pour les préparatifs culinaires². L'image de l'intérieur d'une cuisine moyenne du XVII e siècle à Venise, se complète d'un mobilier sommaire mais utilitaire, tel que des chaises en osier de qualité modeste. Les planches en bois répondent à la nécessité de préparer, poser ou traiter les aliments divers ${ }^{3}$. Outre les planches, on répertorie aussi des tables à tréteaux. Quant aux meubles de rangement où l'on dispose vaisselle et le linge cuisine, ils se présentent, soit sous forme de niches aménagées dans les murs, soit sous forme de dressoirs mobiles. On pourrait citer à titre d'exemple, la demeure de Ioannis Matheos Agapitos (1655) ${ }^{4}$ où on a enregistré un dressoir oblong en bois de sapin ayant la forme de credenza (un armer d'albeo lungo da cusina in forma di credenza), la crédence étant le meuble par excellence pour ranger et exposer la vaisselle d'apparat. Il est donc évident que la diversité des biens mobiliers et des denrées de la représentation générale de la cuisine, comme par exemple la présence de pots de conservation et de coffres contenant une certaine quantité de produits, tels que l'huile ou la farine, attestent de l'aisance matérielle du maitre des lieux. La vaisselle, les ustensiles ainsi que les provisions en vivres, étaient conservés dans des coffres de plus grande capacité appelés casson, cassone ou cassoni.

4 La prise des repas se faisait soit dans la cuisine soit, ce qui est d'usage, dans une pièce voisine qui tenait lieu de salle à manger et où l'on trouve une grande table et un certain nombre de chaises. La cuisine donc, en tant qu'espace de rangement, de préparation et éventuellement de prise des repas aussi bien que la salle à manger, destinée aux déjeuners et aux dîners, sont les deux piliers fondamentaux autour desquels se regroupent famille, hôtes de passage et domestiques (GRIECO, 2006, 244-252; CURRIE, 2006, 20-43 ; SARTI, 2000, 171-224).

5 Nous allons examiner plus en détail l'inventaire après décès daté de 1648 , de Epiphanios Igoumenos ${ }^{5}$, riche commerçant, originaire de Ioannina, en Épire, et président de la confrérie grecque de Venise (merTzios, 1965, 107-111). Dans ce témoignage, est enregistré, non seulement la cuisine richement équipée (BLAKE, 2006, $332-339,341$ ), mais aussi l'office du panetier ; in un luogo da far pan, à savoir l'espace qui sert à fabriquer du pain et dans lequel étaient réunis tous les ustensiles et matériaux 
nécessaires dont un vieux réchaud en cuivre, caldiera, destiné à laisser lever la pâte au chaud avant la cuisson. Également recensés, un coffre pour conserver la farine, à moitié plein de farine (mezzo star di farina), deux gourdes en bois (fiasche), récipients pour la fabrication de pain, deux passoirs et un tamis à farine (tamisi e burataora) tous usés, une scie, une aiguière et bassin à laver les mains (cadini e brocca), un panier à verre (una cesta de veri), et une boîte à cendres en bois de sapin (una cassa da cenere d'albeo).

Dans la cuisine d'Epiphanios Igoumenos, outre les trois vieilles chaises en osier, aucun autre meuble n'a été enregistré. Il y avait cependant un grand nombre de matériel de cuisine, désignés comme vieux (vecchi) et usés (usadi). Parmi eux, on trouve sept seaux en bronze, un récipient appelé rifrescadora - c'est à-dire un rafraichisseur pour préserver les liquides et les aliments à basse température -, quatre chauffe-lits, des cuillères, un réchaud, nommés alors "chauffoir ou réchauffoir " pour maintenir les aliments au chaud (scalda vivanda), des poêles, quatre chaudrons, ainsi que tous les ustensiles complémentaires en fer pour la cheminée, voire deux trépieds, du matériel de fer pour faire du feu (cavioni), et un crochet (rampegonzin). Sont aussi recensés : un pose savon en cuivre et un bassin en laiton avec l'aiguière pour se laver les mains. Pour l'éclairage de la pièce étaient utilisées cinq lampes à huile en laiton et quatre petits chandeliers. Enfin, deux vaisselles de table dont le nombre de plats est impressionnant, l'une, en étain comptait cent onze assiettes, de petit et de grand format, et l'autre cent six.

7 Une source d'information importante pour l'organisation de la cuisine d'une demeure aristocratique est l'inventaire des biens mobiliers, daté de 1683, de Niccolò Kalergi' ${ }^{6}$, gentilhomme créto-venitien. L'inventaire cuisine commence avec la phrase «suivent les bronzes et le matériel de cuisine » (seguono i rami e robbe di cucina), et répertorie une très riche liste de moyens utilitaires. Les deux fourneaux de bronze (forni) avec leurs couvercles figurent parmi les objets les plus rares et par conséquent les plus précieux. On suppose qu'il y avait possibilité de préparer de grands repas de réception, en cuisson simultanée. Parmi les ustensiles recensés il y en a qui sont dignes de mention, ainsi dix trépieds en métal pour supporter chaudrons, marmites ou poêles, un pot avec manche en bronze qui servait à conserver le gras (lecarda), cinq petites et grandes louches (cazza), deux râpes (grattacassa), deux chauffe-lits (scaldaletti), un petit couperet en forme de hache (manara), une balance (belanza), un moule pour la décoration de friandises aux raisins et aux épices (stampo dà mustazzoni). Pour l'éclairage, on se servait de deux grandes lanternes, les ferale, et de quatre chandeliers en bronze. Ce qui manque à cet inventaire de la cuisine de Kalergi c'est la vaisselle et les couverts, considérés comme des objets de luxe et souvent gardés séparément. Surtout les couteaux et les fourchettes en argent ou à manche en ivoire et argent, des biens personnels favoris du maître, étaient conservés dans des étuis en cuir, en tissu ou dans de petits coffrets.

Outre les témoignages d'archives, les sources iconographiques, telles que la peinture et les gravures, nous permettent de mieux restituer cet espace modeste. Le grand nombre de peintures illustrant avec réalisme des scènes de cuisine et par extension les pratiques alimentaires au XVII ${ }^{\mathrm{e}}$ siècle, témoignent de l'importance accordée aux objets utilitaires $^{7}$. La représentation iconographique des repas par les peintres post-byzantins tel que les Noces de Cana ${ }^{8}$, sujet inspiré par les Saintes Écritures, éclaire bien qu'indirectement, les aspects de la culture matérielle de l'époque liés à la nutrition.

Les Grecs de Venise au XVII ${ }^{e}$ siècle, constituent un ensemble ethno-religieux défini par des traits communs comme l'adaptabilité et la volonté d'intégration dans la société 
vénitienne, au moins en ce qui concerne les questions pratiques de la vie quotidienne. Quant à l'organisation de l'espace cuisine notamment, et selon les sources iconographiques et les témoignages d'archive, tels que celui publié et édité par Isabella Palumbo Fossati Casa pour le Xvi ${ }^{\mathrm{e}}$ siècle (2013, 49-51, 83-84, 176, 184-185), le choix et l'usage du matériel utilitaire quotidien s'apparente à celui que l'on rencontre dans toutes les demeures vénitiennes de l'époque. Bien que la majorité du matériel cuisine ne présente pas de différences en termes de forme, de matériau et d'utilité par rapport à ceux utilisés en Grèce, on rencontre pourtant certains ustensiles plus élaborés.

Il est évident que les formes fixes des objets cuisine quotidiens, étroitement liées aux habitudes gastronomiques grecque-vénitiennes, très proches les unes aux autres, sont formées par les interactions et les échanges au cours d'une longue cohabitation (VLASSI, 1998, 56-75 ; VLASSI, 2010, 366-373 ; VARZELIOTI, 2015, infra). Il s'agit d'une riche culture alimentaire commune, autant en termes de stockage, de traitement et de préparation d'aliments ainsi qu'en termes d'adoption et d'utilisation de biens mobiliers qui indiquent une culture matérielle et une pratique nutritionnelle identiques. Ainsi, les objets répertoriés dans les foyers grecs de Venise, manifestent à la fois les choix culinaires ainsi que le rang social des propriétaires.

11 Par ailleurs, la vaisselle et les couverts d'apparat gardés dans des coffres, des dressoirs, des crédences ou des paniers, souvent dans des pièces autres que la cuisine, attestent de l'aisance matérielle, définissent l'esthétique du goût du maître et s'alignent sur les pratiques de consommation courantes de l'époque. Enfin, la séparation de la cuisine de la salle à manger dans les demeures des Grecs de Venise, en particulier celles des plus prospères, s'inscrit dans le cadre des coutumes urbaines vénitiennes aussi bien que celui des centres urbains européens (QUELLIER, 2013, p.99-111) ${ }^{9}$. Tous ces éléments confèrent à l'étude de la culture matérielle et des objets associés à l'alimentation des Grecs de Venise et nous permettent d'établir des preuves quant à leur mode culinaire et de faire des enquêtes comparatistes avec d'autres sources provenant de témoignages d'archives, littéraires et iconographiques.

\section{BIBLIOGRAPHIE}

AJMAR-WOLLHEIM Marta \& DENNIS Flora (dir.), 2006, At Home in Renaissance Italy, Londres: V\&A Museum.

AJMAR-WOLLHEIM Marta, 2006, “Sociability”, in Marta AJMAR-WOLLHEIM \& Flora DENNIS (dir.), op. cit., pp. 206-214.

BLAKE Hugo, 2006, "Everyday objects”, in Marta AJMAR-WOLlHeIM \& Flora DENNIS (dir.), op. cit., pp. 332-339, 341.

CECCHINI Isabella, 2000, Quadri e commercio a Venezia durante il Seicento: uno studio sul mercato dell'arte [Tableaux et commerce à Venise durant le XVII ${ }^{e}$ siècle : une étude du marché de l'art], Venise : Saggi Marsilio. 
CURRIE Elisabeth, 2006, "The reception room and kitchen: the social life of the family", in Inside the Renaissance House, Londres : V\&A Publications, pp. 20-43.

FORTINI BROWN Patricia, 2005, Private Lives in Renaissance Venice. Art, Architecture and the Family, New Haven-Londres : Yale University Press.

GRIECO Allen J., 2006, “Meals”, in Marta AJMAR-WOLLheIM \& Flora DENNIS (dir.), op. cit., pp. 244-252. HECK Michèle-Caroline (dir.), 1997, Sébastien Stoskopff, 1597-1657 : un maître de la nature morte. Musée de l'Euvre Notre-Dame, Strasbourg, 15 mars-15 juin 1997, Strasbourg : Éditions de la Réunion des musées nationaux.

LATRÉMOLIÈRE Élisabeth \& QUELLIER Florent (dir.), 2012, Festins de la Renaissance : cuisine et trésors de la table, Paris : Somogy éditions d'art - Musée du Château royal de Blois.

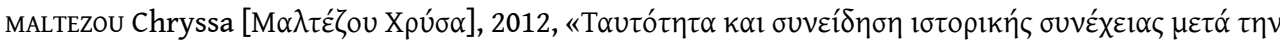

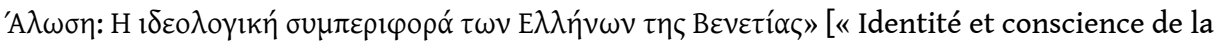

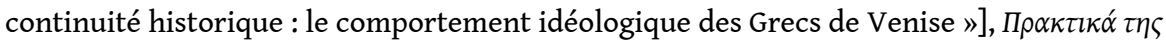

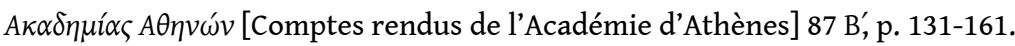

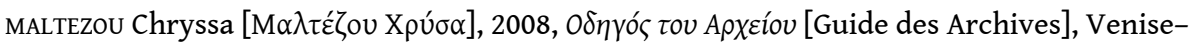
Athènes : Institut hellénique d'études byzantines et post-byzantines de Venise.

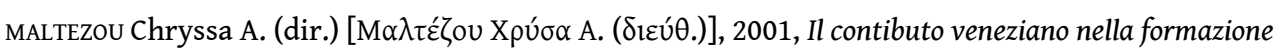
del gusto dei Greci (XV-XVII sec.), Atti del Convegno Internazionale, Venezia, 2-3 giugno 2000 [La contribution vénitienne à la formation du goût des Grecs (XV-XVII ${ }^{\mathrm{e}}$ siècles), Actes du Congrès international, Venise, 2-3 juin 2000], Venise : Institut hellénique d'études byzantines et postbyzantines de Venise.

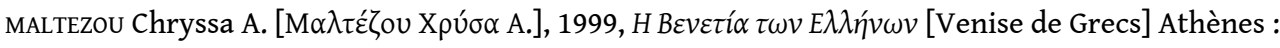
Militos.

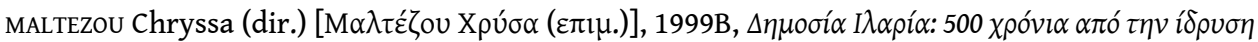

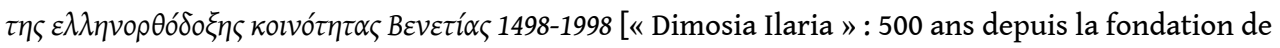
la communauté grecque-orthodoxe de Venise 1498-1998], Venise : Institut hellénique d'études byzantines et post-byzantines de Venise.

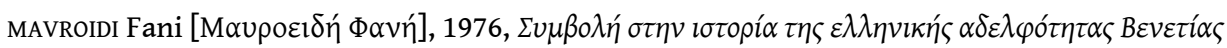

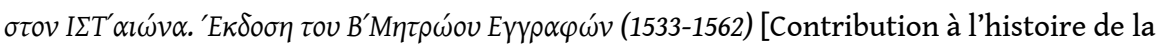
Confrérie grecque de Venise au XVI ${ }^{\mathrm{e}}$ siècle. Publication du Registre B'des inscriptions des confrères (1533-1562)], Athènes.

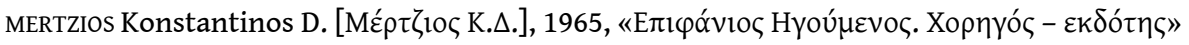

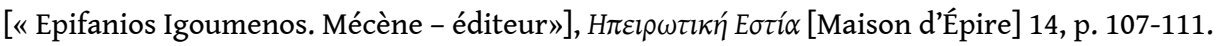
QUELLIER Florent, 2013, la Table des Français : une histoire culturelle (XVe-début XIXe siècle), Rennes : Presses universitaires de Rennes - Presses universitaires François Rabelais.

PALUMBO FOSSATI CASA Isabella, 2013, Dentro le case: Abitare a Venezia nel Cinquecento [Intérieurs vénitiens à la Renaissance], Venise : Gambier \& Keller éditions.

PALUMBO FOSSATI Isabella, 1984, «L'interno della casa dell'artigiano e dell'artista nella Venezia del Cinquecento» [ « L'intérieur de la maison d'artisan et d'artiste à Venise au XVI ${ }^{\mathrm{e}}$ siècle »], Studi Veneziani 8, p. 109-153.

SARTI Raffaella, 2006, Vita di casa: Abitare, mangiare, vestire nell'Europa moderna [la Vie à la maison : habiter, manger, habiller à l'Europe moderne], Rome-Bari : Laterza, p. 171-224. 
scully Terence (trad.), 2008, 1570 The Opera of Bartolomeo Scappi (1570): The Art and Craft of a Master Cook [l'CEuvre de Bartolomeno Scappi], traduite et commentée par Terence Scully, Toronto : University of Toronto Press.

SCHUURMAN Anton J., 1980, «Gli inventari “post mortem” come fonte per lo studio della cultura materiale: Un programma olandese di ricerca» [ « Les inventaires “après décès" come source pour l'étude de la culture matérielle : un programme de recherche hollandais »], Quaderni Storici [Cahiers Historiques] 43, p. 210-218.

TRIANTAFYLLIDOU-BALADIÉ Yolande, 1986, « Les objets de première nécessité et les signes distinctifs de richesse dans le mode de vie de la société ottomane en Crète aux XVII ${ }^{\mathrm{e}}$ et XVIII ${ }^{\mathrm{e}}$ siècles ", in Inventaires après-décès et ventes de meubles : apport à une histoire de la vie économique et quotidienne, XIV ${ }^{e}$ XIX ${ }^{e}$ siècles, Congrès international d'histoire économique, M. BAULANT \& A. J. SCHUURMAN (dir.), Berne : P. Servais, p. 347-359.

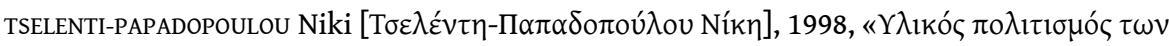

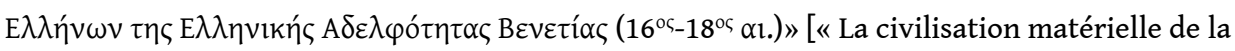

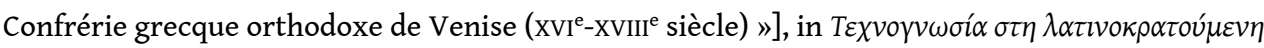
$E \lambda \lambda \alpha ́ \delta \alpha$ [Expertise en Grèce durant la période de la domination latine], Athènes : Fondation culturelle de la Banque du Pirée, p. 89-95.

TSELENTI-PAPADOPOULOU Niki, 2004, « Les communautés grecques en Italie (XVe-XVIII ${ }^{\mathrm{e}}$ siècles) à partir

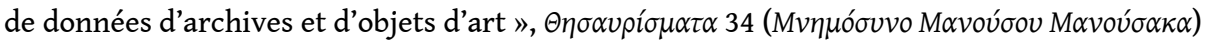
[Thesaurismata 34 (In memoriam Manousos Manousakas)], Revue de l'Institut hellénique d'études byzantines et post-byzantines de Venise, p. 459-471.

VAN DER WOUDE Adrianus \& SCHUURMAN Anton (dir.), 1980, Probate inventories: A new source for the historical study of wealth, material culture and agriculture development [Inventaires homologués : une nouvelle source pour l'étude historique de la richesse, de la culture matérielle et du développement agricole], Papiers présentés au "Leeuwenborch Conference" (Wageningen, 5-7 mai 1980), Utrecht: Hes Publishers.

VARZELIOTI Georgia, 2015, « Le repas de noces dans la Crète de la Vénétocratie : de nouvelles informations sur l'histoire de la nourriture à Candie au XVII ${ }^{e}$ siècle ", in les Actes du XXIV ${ }^{e}$ Congrès pluridisciplinaire des néo-hellénistes francophones, Irini TSAMADOU-JACOBERGER \& Martine BREUILLOT (dir.), Manger en Grèce, Université de Strasbourg, 27-29 mai 2015, Strasbourg : Université de Strasbourg.

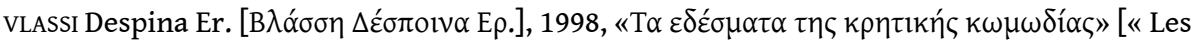

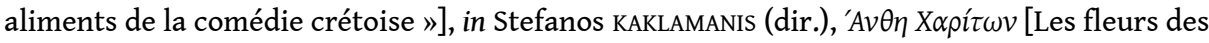
grâces], Venise : Institut hellénique d'études byzantines et post-byzantines de Venise, p. 56-75.

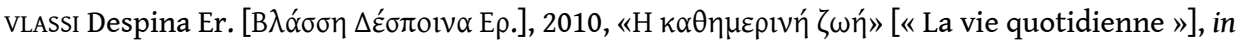

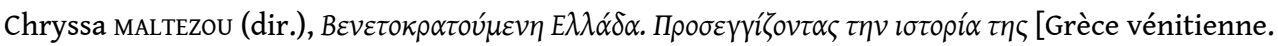
Aborder l'histoire], vol. A, Athènes-Venise : Institut hellénique d'études byzantines et postbyzantines de Venise, p. 366-373.

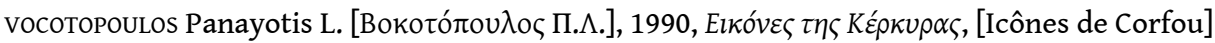
Athènes : Fondation Culturelle de la Banque Nationale de la Grèce (MIET).

ZANATA Francesca, 1997, «L'inventario come fonte per lo studio della storia della ricchezza privata: Venezia nel 1661» [ « L'inventaire comme source pour l'étude de l'histoire de la richesse privée : Venise à l'an 1661 », Studi Veneziani 34, p. 199-223. 


\section{NOTES}

1. La bibliographie pour les inventaires après décès est très riche. Voir à titre indicatif en ordre chronologique : SCHUURMAN, 1980, p. 210-218; VAN DER WOUDE \& SCHUURMAN (dir.) 1980 ; PALUMBO FOSSATI, 1984, p. 109-153 ; TRIANTAFYLLIDOU-BALADIÉ, 1986, p. 347-359 ; ZANATA, 1997, p. 199-223 ; CECCHINI, 2000 ; PALUMBO FOSSATI CASA, A2013.

2. Par exemple pour la cheminée, on peut consulter les gravures qui illustrent l'œuvre $\mathrm{du}$ Bartolomeo Scappi, cuisinier fameux du $\mathrm{xvI}^{\mathrm{e}}$ siècle. Ces gravures sont caractéristiques pour la formation d'une cuisine typique et assez prospère de l'époque, et en plus pour les ustensiles de tout type : l'Euvre du Bartolomeno Scappi (1570). L'art et métier d'un cuisinier maître, 2008, p. 636-662, ill. 1-27.

3. Voir, les planches d'une cuisine représentées dans l'œuvre du peintre Sébastien Stoskopff, les Quatre éléments, ou l'Hiver (vers 1633), exposé au Musée de l'œuvre Notre-Dame de Strasbourg (inv. Mba 2082), нЕск (dir.) 1997, p. 164-166, cat. $n^{\circ} 17$.

4. Archives d'État de Venise, Giudici di Petizion/Inventari, b. 365/29 (20 janvier 1655).

5. Archives d'État de Venise, Notarile Atti (Simone Porta), b. 11026, ff. 32v-40v (20 mai 1648).

6. Archives d'État de Venise, Notarile Testamenti (Francesco Velano), b. 1049, testament $n^{\circ} 214$ (4 novembre 1683).

7. Voir le tableau du Vincenzo Campi, Cuisine ( 1580 c.), peinture très vivante pour les travaux cuisiniers et les habitudes alimentaires, maintenant à l'Académie de Brera, Milan ( ${ }^{\circ} 297$ ) : AJMAR-WOLLHEIM \& DENNIS (dir.) 2006, p. 333, $\mathrm{n}^{\circ}$ de catalogue 87.

8. Comme, par exemple, le tableau Noces de Cana (1561-1570), aujourd'hui au Musée Correr de Venise, qui est attribué à Michel Damaskinos, peintre fameux d'origine crétoise : AJMAR-WOLLHEIM \& DENNIS (dir.), 2006, p. 245, nº de catalogue 50. Voir, en plus, une autre scène de festin de noces au tableau post-byzantin de Stéphane Giancarolas, Saint Alexis, homme de Dieu et des scènes de sa vie (vers 1700), qui est exposé au Musée d'art byzantin d'Antivouniotissa à Corfou: vocotopoulos, 1990, p.160-161, n ${ }^{\circ} \mathrm{de}$ catalogue 128, ill. 62 .

9. Pour la séparation de la cuisine de la salle à manger, voir, par exemple, un tableau caractéristique d'un peintre anonyme de Flandres qui représente le Christ avec les pèlerins d'Emmaüs (fin du XVI ${ }^{\mathrm{e}}$-début du XVII $\mathrm{e}$.), aujourd'hui au palais des Beaux-Arts à Lille, inv.P1547 : LATRÉMOLIÈrE et QUELLIER (dir.) 2012, p. 166, nº 61.

\section{RÉSUMÉS}

Ustensiles de cuisine, de rangement et de service, enregistrés dans les foyers grecs de Venise au $\mathrm{XVII}^{\mathrm{e}}$ siècle, tel est le sujet traité dans le présent article. L'étude de deux groupes importants de documents notariés, les testaments et les inventaires après décès (post-mortem inventari), registres détaillés des biens mobiliers de testateurs grecs, révèlent des aspects de la vie 
quotidienne et de la culture matérielle, quant aux objets appropriés pour le stockage des denrées ainsi qu'à la préparation et la prise de repas. L'étude porte d'une part, sur l'usage diachronique des ustensiles utilitaires simples en termes de forme, de matériau et d'utilité, et d'autre part, sur l'évolution des objets d'apparat, associés au développement du savoir-faire. La recherche d'archives se complète par l'étude des sources iconographiques de peintures et d'autres œuvres artistiques créées par des artistes occidentaux aussi bien que post-byzantins, représentant des scènes de cuisine et les pratiques alimentaires liées au XVII ${ }^{\mathrm{e}}$ siècle.

This paper attempts to study the utensils for cooking, storage and serving of food as they have been recorded in Greek households of the 17th century Venice. Studying the two most important groups of notary papers, the wills and the post-mortem inventari, i.e. the recording of movable property after the death of Greek testators, uncovers aspects of daily life and the material culture related to objects deemed appropriate for the storage, preparation and serving of food. We follow the daily use of plain utensils according to shape, material and use, and the development of luxury objects related to various forms of socialization. Archival research is furthermore complemented with the study of paintaings and depictions of the kitchen and dining areas by Western as well as post-Byzantine artists.

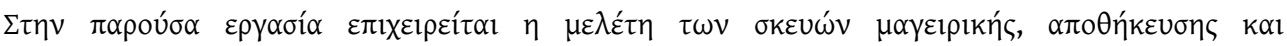

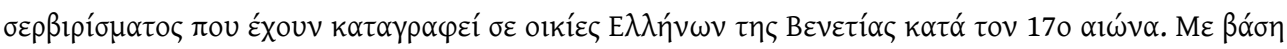

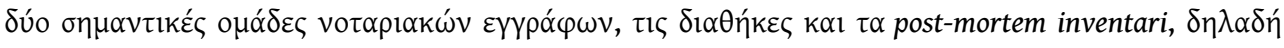

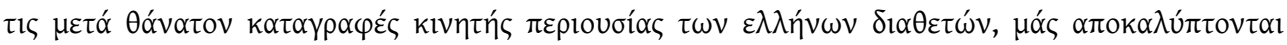

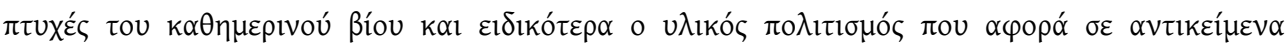

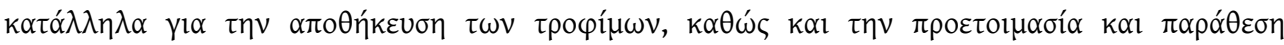

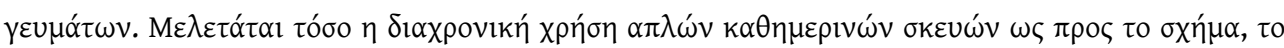

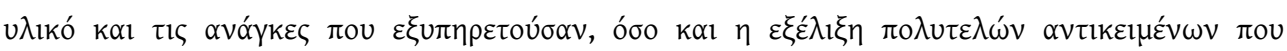

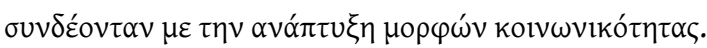

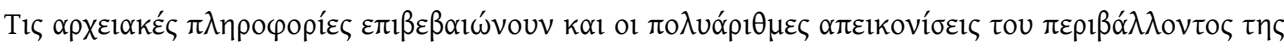

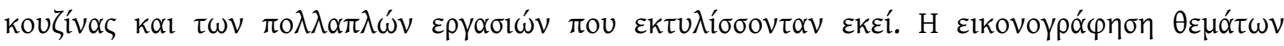

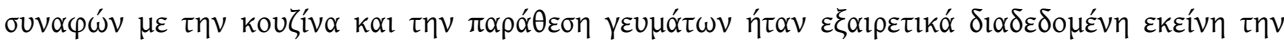

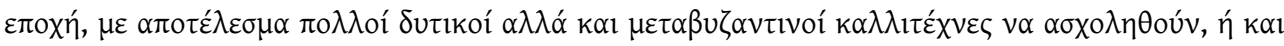

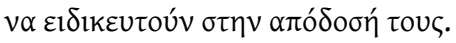

\section{INDEX}

motsclestr Gastronomi, İkonografi, Yunan diasporası, Venedik, On yedinci yüzyıl, Sosyal tarih

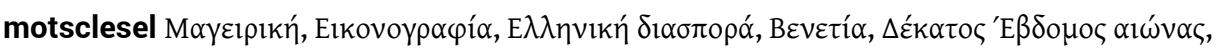

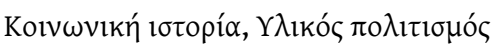

motsclesmk ГАСТРОНОМИЈАТА, ИКОНОГРАФИЈА, ГРЧКАТА ДИЈАСПОРА, ВЕНЕЦИЈА, СЕДУМНАЕСЕТТИ ВЕК, СОЦИЈАЛНА ИСТОРИЈА

Mots-clés : cuisine, cuisine, iconographie, iconographie, diaspora grecque, diaspora grecque, culture matérielle, culture matérielle, Grecs de Venise, Grecs de Venise

Keywords : Cooking, Iconography, Greek Diaspora, Venice, Seventeenth century, Social History, Material Culture

Thèmes : Histoire sociale

Index géographique : Venise

Index chronologique : dix-septième siècle 
AUTEUR

OURANIA KARAGIANNI

docteure en archéologie et histoire de l'art byzantin - Université d'Athènes 